\title{
A modal method for finite amplitude, nonlinear sloshing
}

\section{P N SHANKAR and R KIDAMBI}

Computational and Theoretical Fluid Dynamics Division. National Aerospace Laboratories, Bangalore 560 017, India

Email: pns@ctfd.cmmacs.ernetin: kidambi@ctfd.cmmacs.ernet.in

MS received 19 February 2002

Abstract. A modal method is used to calculate the two-dimensional sloshing motion of an inviscid liquid in a rectangular container. The full nonlinear problem is reduced to the solution of a system of nonlinear ordinary differential equations for the time varying coefficients in the expansions of the interface and the potential. The effects of capillarity are included in the formulation. The simplicity, generality and power of the method are exhibited not only by recovering the earlier results obtained, for example, by Penney and Price [1], Tadjbakhsh and Keller [2] and Faltinsen et at [3], but also by obtaining new and interesting results of the effects of capillarity and shallow depth, which would be difficult to obtain otherwise. For example, it is found that for the initial interface profile considered here, parasitic capillary waves, borne by the higher number wave modes, are generated for moderate capillarity but disapp
simple geometries.

Keywords. Nonlinear sloshing; modal method; capillarity

\section{PACS Nos $47.35 .+i ; 47.11 .+j ; 47.15 . H g$}

\section{Introduction}

We are concerned in this paper with the irrotational sloshing motion of an inviscid, incompressible fluid in a rectangular container. The motion is assumed to be two-dimensional. All lengths are normalized by $L$, the width of the container, time by $\sqrt{L / g}$, velocities by $\sqrt{g L}$, acceleration by $g$, the potential by $L \sqrt{g L}$ and the pressure by $\rho g L$. We shall take into consideration the effects of capillarity. In what follows, the surface tension $\sigma$ appears nondimensionally as the parameter $\beta=\sigma / \rho g L^{2}$. If the surface of the liquid is assumed to be given by $y(x, t)=\eta(x, t)$ and the coordinate system is as shown in figure 1 fixed to the possibly moving container, the governing equations and boundary conditions can be written down as follows:

$$
\mathbf{u}(x, y, t)=\nabla \phi
$$

$\nabla^{2} \phi(\mathbf{x}, t)=0$ in $\mathscr{D}=\{-0.5<x<0.5,-h<y<\eta(x, t)\}$ 


$$
\phi_{x}=0 \quad \text { on } \quad x= \pm 0.5 ; \phi_{y}=0 \quad \text { on } \quad y=-h
$$

$$
\left.\eta_{t}+u \eta_{x}=v \quad \text { on } y=\eta(x, t)\right\}
$$

$$
\phi_{t}+\frac{\left(u^{2}+v^{2}\right)}{2}+\eta\left(1+A_{y}(t)\right)+x A_{x}(t)=\beta \frac{\eta_{x x}}{\left(1+\eta_{x}^{2}\right)^{3 / 2}}
$$

$$
\text { on } y=\eta(x, t) \text {. }
$$

Equations (1a)-(Id) assert respectively that the velocity is derivable from a potential, that the potential is harmonic, that the normal velocity on the sidewalls and the bottom wall at $y=-h$ vanish. The boundary condition ( $1 \mathrm{~d})$ on the interface $y=\eta(x, t)$ ensures the integrity of the interface as it changes with time while (le) enforces the Young-Laplace surface tension condition with the help of the unsteady Bernoulli equation. $A_{x}(t)$ and $A_{y}(t)$ are the arbitrary linear accelerations that the container may undergo in the $x$ and $y$ directions. $\beta=\sigma / \rho g L^{2}$ is the inverse of the Bond number, $B o$. These equations and conditions will have to be supplemented with initial conditions on the interface and the field.

It is necessary, since we are including the effects of capillarity, to consider what happens at the point where the liquid surface meets the solid side wall. In the static case the notion that there is a well-defined and unique contact angle for a given liquid, gas and solid triplet seems well founded. However, the dynamic case seems to be far less certain (see Dussan [4]). Moreover, there seem to be theoretical difficulties in handling the contact angle condition and as a consequence most authors either fix the contact angle at some value, or ignore it or pin the interface edge (see Hocking [5]). We too have not been able to satisfactorily resolve this issue. Our position is that the following analysis is exactly true for a contact angle of $\pi / 2$ in the symmetric case and we believe this restriction can be lifted in this case. However, in the general asymmetric and nonsymmetric case we shall not impose a contact angle condition. Although we have no proof of this, we believe that unless the container is very narrow the contact angle is unlikely to seriously affect the primary phenomena that are of interest to us.

We note four sources of nonlinearity: (a) The advection term $u \eta_{x}$ in the interface boundary condition to ensure the integrity of the boundary, (b) the kinetic energy term in the pressure boundary condition on the interface, (c) the curvature term involving $\beta$

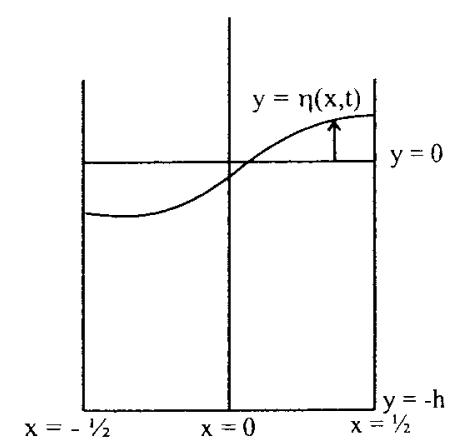

Figure 1. Sloshing of an inviscid liquid in a rectangular container of unit width. The undisturbed liquid is of depth $h$, while $\eta(x, t)$ is the elevation of the liquid surface from the mean level $y=0$. in the pressure boundary condition on the interface (d) the domain in which the problem has to be solved is changing and has to be determined as a part of the solution.

There is a large body of literature dealing with the problem as posed above and with the related classical problem of wave motion in an unbounded ideal fluid. For the important linearized analysis one can refer to Lamb [6]; and to Stokes $[/]$ and Penney and Price $\{1\}$ for the analysis of finite amplitude periodic progressive and periodic stationary waves respectively. Tadjbakhsh and Keller [2] studied the effect of finite depth on periodic stationary waves while Concus $[8]$ extended their work 10 include the effects of surface tension. Schwartz and Whitney [9] corrected and improved Penney and Price [1] to 25th order. An exact solution for two-dimensional progressive waves of arbitrary amplitude on a fluid of infinite depth, when the only restoring force is surface tension, was found by Crapper [10]. A number of analytical and numerical studies exist on liquid sloshing in containers undergoing horizontal, vertical and pitching motions but mostly in simple rectangular, cylindrical and spherical geometries. We content ourselves with providing a few references ([11-16]). Moore and Perko [17], Perko [18], Faltinsen et al [3] and La Rocca et al [19] are some of the works that are closest in spirit to the present approach. While all of them employ modal approaches, there are significant differences in the ways these are derived and used. In [17] and most of [18], the interface is not defined analytically in terms of a spatial coordinate. Also, we are able to handle large curvatures and large surface tension which these works are unable to. References [3] and [19] employ variational principles to reduce the interface boundary conditions to an infinite system of ODE's for the unknown coefficients of the generalized Fourier series that they assume for $\eta$ and $\phi$. In [3], a truncated nonlinear system for just the first three modes is used to study sloshing in a linearly accelerated and a pitching container. An assumption in obtaining this system is that the first mode is the dominant one. Though this method is good enough to describe certain aspects of large amplitude sloshing, it has difficulties when the first mode is not the (only) dominant one. This happens, for example, in shallow water or when the initial data contains higher modes. Also, the work as it stands does not incorporate surface tension effects. Thus, although it can handle large amplitude waves, it cannot capture some of the finer features like parasitic capillary waves which are generated on steep gravity waves.

The present work was motivated by the need to have a simple and efficient computational procedure capable of dealing with finite amplitude sloshing in simple geometries, at least. Although the modal approach is well known and has been used, its use in the nonlinear case seems to have been restricted, by and large, to generating higher-order approximations to linearized solutions. The position we take here is that (i) the linear structure and the related modes and (ii) the fact that all the relevant functions must have Fourier series in $x$ for each instant in time, must have a strong bearing on the finite amplitude nonlinear motion. This position leads us to reducing the problem to a system of nonlinear ordinary differential equations $(7 \mathrm{a}, \mathrm{b})$ and $(8 \mathrm{a}, \mathrm{b})$ for unknown functions of time, which we believe is new. We are, with this comparatively simple system, able to solve, fairly easily problems that have posed difficulties in the past. This is first illustrated in $\$ 3$ for initial value problems in a stationary container where we demonstrate the generation of capillary waves when the initial amplitude is large enough; in $\S 4$ for almost periodic sloshing where we compare our results with those of Penney and Price [1] and Tadjbakhsh and Keller [2]; and finally in §5 for sloshing in a linearly accelerating container. 


\section{A modal approach to nonlinear sloshing}

Noting that (i) the linearized solition is a sum of modes, (ii) the potential satisfies a linear equation and has a simple representation in this geometry and (iii) all the field quantitics must have Fourier series representations in $x$ at each instant of time, we seek a solution having the following representation

$$
\begin{aligned}
& \eta(x, t)=\sum_{j=1}^{\infty}\left[a_{j}(t) \cos k_{j} x+c_{j}(t) \sin l_{j} x\right] \\
& \phi(x, y, t)= b_{0}(t)+\sum_{j=1}^{\infty}\left[b_{j}(t) \cos k_{j} x\left\{\frac{\cosh k_{j}(y+h)}{\sinh k_{j} h}\right\}\right. \\
&\left.+d_{j}(t) \sin l_{j} x\left\{\frac{\cosh l_{j}(y+h)}{\sinh l_{j} h}\right\}\right]
\end{aligned}
$$

where $k_{j}=2 j \pi, l_{j}=(2 j-1) \pi, j=1,2,3, \ldots$. As indicated in figure $1, y=0$ has been taken to coincide with the mean level of the liquid surface and as a consequence the mean value of $\eta$ has to be zero at all times. This is why the term $a_{0}(t)$ has not been included in the expansion in (2a). However, in our calculations this mean value is computed as a measure of the accuracy of the computation. On the other hand, the term $b_{0}(t)$ in $(2 \mathrm{~b})$ is essential for the computation of the pressure, even though it does not affect the velocity field. Note that with the above representation the field equations and the sidewall and bottom wall conditions are satisfied automatically. We only need to satisfy the two interface conditions $(1 \mathrm{~d}, \mathrm{e})$ on the unknown interface $y=\eta(x, t)$. However, let us first write down the expressions for the velocity components in the liquid

$$
\begin{aligned}
u(x, y, t)= & \phi_{x}=\sum_{j=1}^{\infty}\left[-k_{j} b_{j}(t) \sin k_{j} x\left\{\frac{\cosh k_{j}(y+h)}{\sinh k_{j} h}\right\}\right. \\
& \left.+l_{j} d_{j}(t) \cos l_{j} x\left\{\frac{\cosh l_{j}(y+h)}{\sinh l_{j} h}\right\}\right] \\
v(x, y, t)= & \phi_{y}=\sum_{j=1}^{\infty}\left[k_{j} b_{j}(t) \cos k_{j} x\left\{\frac{\sinh k_{j}(y+h)}{\sinh k_{j} h}\right\}\right. \\
& \left.+l_{j} d_{j}(t) \sin l_{j} x\left\{\frac{\sinh l_{j}(y+h)}{\sinh l_{j} h}\right\}\right] .
\end{aligned}
$$

It may be observed that the terms expressing the $y$-dependence contain a $\sinh k h$ or $\sinh l h$ in the denominator. While this is not essential, it helps to both reduce the above expressions to the corresponding ones for the infinitely deep cavity when $h \rightarrow \infty$ and to significantly reduce the size of certain matrix elements that have to be calculated later

We are now ready to derive the main equations used in this paper. First, guided by the procedure used to derive the linearized solution to the problem, write $(l e, f)$ as follows:

$$
\begin{aligned}
\eta_{i t}=v^{\prime}(x, \eta, t)-u(x, \eta, t) \eta_{i} \\
\phi_{i}(x, \eta, t)=-\eta \eta+\beta \eta_{x . t}-\frac{\left(u(x, \eta, t)^{2}+v^{\prime}(x, \eta, t)^{2}\right\}}{2} \\
+\beta \frac{\eta_{x x}\left\{1-\left(1+\eta_{s}^{2}\right)^{3 / 2}\right\}}{\left(1+\eta_{x}^{2}\right)^{3 / 2}} .
\end{aligned}
$$

Now, since the above are to hold for all $t>0$ on $-1 / 2<x<1 / 2$, the Fourier coefficients of each side must equal those of the other side for all positive $t$. This suggests that valid equalities will be obtained by multiplying each equation by $\cos k_{j} x$ and $\sin l_{j} x$ and integrating from $-1 / 2$ to $1 / 2$. Let us first define the following integrals:

$$
\begin{aligned}
& \mu_{i j}^{\prime}(t)=k_{j} \int_{-1 / 2}^{1 / 2} \cos k_{i} x \cos k_{j} x\left\{\frac{\sinh k_{j}(\eta+h)}{\sinh k_{j} h}\right\} \mathrm{d} x \\
& \mu_{i j}^{2}(t)=l_{j} \int_{-1 / 2}^{1 / 2} \cos k_{i} x \sin l_{j} x\left\{\frac{\sinh l_{j}(\eta+h)}{\sinh l_{j} h}\right\} \mathrm{d} x \\
& \xi_{i}^{\prime}(t)=-\int_{-1 / 2}^{1 / 2} \cos k_{i} x u(x, \eta, t) \eta_{x} \mathrm{~d} x \\
& \gamma_{i j}^{1}(t)=l_{j} \int_{-1 / 2}^{1 / 2} \sin l_{i} x \sin l_{j} x\left\{\frac{\sinh l_{j}(\eta+h)}{\sinh l_{j} h}\right\} \mathrm{d} x \\
& \gamma_{i j}^{2}(t)=k_{j} \int_{-1 / 2}^{1 / 2} \sin l_{i} x \cos k_{j} x\left\{\frac{\sinh k_{j}(\eta+h)}{\sinh k_{j} h}\right\} \mathrm{d} x \\
& \xi_{i}^{2}(t)=-\int_{-1 / 2}^{1 / 2} \sin l_{i} x u(x, \eta, t) \eta_{x} \mathrm{~d} x \\
& v_{i j}^{1}(t)=\int_{-1 / 2}^{1 / 2} \cos k_{i} x \cos k_{j} x\left\{\frac{\cosh k_{j}(\eta+h)}{\sinh k_{j} h}\right\} \mathrm{d} x \\
& v_{i j}^{2}(t)=\int_{-1 / 2}^{1 / 2} \cos k_{i} x \sin l_{j} x\left\{\frac{\cosh l_{j}(\eta+h)}{\sinh l_{j} h}\right\} \mathrm{d} x \\
& \chi_{i}^{1}(t)=\int_{-1 / 2}^{1 / 2} \cos k_{i} x\left[-\frac{u^{2}+v^{2}}{2}+\beta \frac{\eta_{x x}\left\{1-\left(1+\eta_{x}^{2}\right)^{3 / 2}\right\}}{\left(1+\eta_{x}^{2}\right)^{3 / 2}}\right] \mathrm{d} x \\
& \delta_{i j}^{1}(t)=\int_{-1 / 2}^{1 / 2} \sin l_{i} x \sin l_{j} x\left\{\frac{\cosh l_{j}(\eta+h)}{\sinh l_{j} h}\right\} \mathrm{d} x \\
& x_{i}^{2}(t)=\int_{-1 / 2}^{1 / 2} \sin l_{i} x\left[-\frac{u^{2}+v^{2}}{2}+\beta \frac{\eta_{x x}\left\{1-\left(1+\eta_{x}^{2}\right)^{3 / 2}\right\}}{\left(1+\eta_{x}^{2}\right)^{3 / 2}}\right] \mathrm{d} x . \\
& \sinh l_{j} h \cos k_{j} x\left\{\frac{\cosh k_{j}(\eta+h)}{1 / 2}\right\} \mathrm{d} x
\end{aligned}
$$

With the above definitions in hand, if we now carry out the programme suggested earlier, namely multiply each of (4) by $\cos k_{j} x$ and $\sin l_{j} x$ and integrate, we obtain the following infinite system of nonlinear ordinary differential equations for the time dependent coefficients $a_{j}(t), b_{j}(t), c_{j}(t)$ and $d_{j}(t)$ in $(2)$. 


$$
\begin{aligned}
& \frac{1}{2} \dot{a}_{i}=\sum_{j}\left[\mu_{i j}^{1} b_{j}+\mu_{i j}^{2} d_{j}\right]+\xi_{i}^{1} \\
& \frac{1}{2} \dot{c}_{i}=\sum_{j}\left[\gamma_{i j}^{2} b_{j}+\gamma_{i j}^{1} d_{j}\right]+\xi_{i}^{2} \\
& \sum_{j}\left[v_{i j}^{1} \dot{b}_{j}+v_{i j}^{2} \dot{d}_{j}\right]=-\frac{1}{2}\left(1+\beta k_{i}^{2}\right) a_{i}+\chi_{i}^{1} \\
& \sum_{j}\left[\delta_{i j}^{2} \dot{b}_{j}+\delta_{i j}^{1} \dot{d}_{j}\right]=-\frac{1}{2}\left(1+\beta l_{i}^{2}\right) c_{i}+\chi_{i}^{2}
\end{aligned}
$$

for $i=1,2,3, \ldots$ It should be noted that so far no approximations have been made and so the above equations are exact.

Naturally, in any actual calculation of finite amplitude sloshing, we will have to truncate the representations (2) to a finite number of terms and give all the coefficients at some time $t=0$, solve the truncated systems (7) and (8) for the finite number of coefficients. We shall take the same number $N$ each of significant coefficients $a_{j}(t), b_{j}(t), c_{j}(t)$ and $d_{j}(t)$; in situations where there is symmetry about the mid-plane, two sets of coefficients will vanish and we will only have to compute for $N$ each of the nonvanishing ones. This is in general not true for antisymmetric data; in this case all four coefficients are required.

The number of terms $N$ that are required to give an accurate representation of the motion depends both on the initial conditions and the amplitude of the motion. For initial disturbances that can be represented by a small number of modes and which are of small amplitude $N$ can satisfactorily be in the range 1-5; otherwise, $N$ will have to be larger. For the examples discussed in this paper $N$ has been in the range 5-20, the large $N$ 's in many cases being used only to check the results.

The system of ODE's (7) and (8) can be solved by any of the standard methods normally used such as the Runge-Kutta methods. Although we have tried these we have found that the implicit methods such as the Adams-Moulton method are less prone to instability; most of the results presented here have been obtained using implicit methods.

\section{Symmetric sloshing in a stationary container}

We begin by considering a simplified situation, one where the container is stationary, where the fluid is initially quiescent and the interface is initially symmetric about the midplane $x=$ 0 . The resulting motion will then be symmetric about this plane and there is considerable simplification in the analysis of the motion. Now, only the expansion coefficients $\left\{a_{j}\right\}$ and $\left\{b_{j}\right\}$ in (2) need to be retained while of the integrals (5) and (6) only $\left\{\mu_{i j}^{1}\right\},\left\{v_{i j}^{1}\right\},\left\{\xi_{i}^{1}\right\}$ and $\left\{\chi_{i}^{1}\right\}$ are significant. Now if $N$ terms are retained there are $2 N$ unknowns to be determined from the $2 N$ coupled ODE's (5a) and (6a).

Through out this section we will assume that the initial shape of the interface is given by $\eta(x, 0)=\eta_{0}(x)=\varepsilon \cos 2 \pi x$, i.e., the initial shape corresponds to the first linearized mode. Then according to the linearized theory, the motion will be a stationary, time periodic motion with the interface given by

$$
\eta_{l}(x, t)=\varepsilon \cos \omega_{l} t \cos 2 \pi x
$$

where $\left(t_{i}=\sqrt{ }\left\{2 \pi\left(1+4 \beta \pi^{2}\right) \tanh 2 \pi h\right\}\right.$ is the linear frequency of this first mode. In figure 2 we compare the results from the linearized theory with those from the present procedure for small amplitudes of motion when we would expect the former to hold. What is shown in the figure are the osciliations in time of the point on the interface in the symmetry plane and we find excellent agreement between the two results. However, a careful look at figure $2 \mathrm{~b}$ shows that even for as small an amplitude as $\varepsilon=0.004$ there are noticeable differences: in figure $2 b$, a discrepancy is evident in every alternate peak leading to a modulation of the basic wave motion by nonlinearity. Naturally, this is even more evident in figure 3 for $\mathcal{E}=0.01$. In figure $3 \mathrm{a}$ the departures from linearity are apparent at both the maxima and the minima of the oscillations. Although subharmonic modulation of the linear wave form
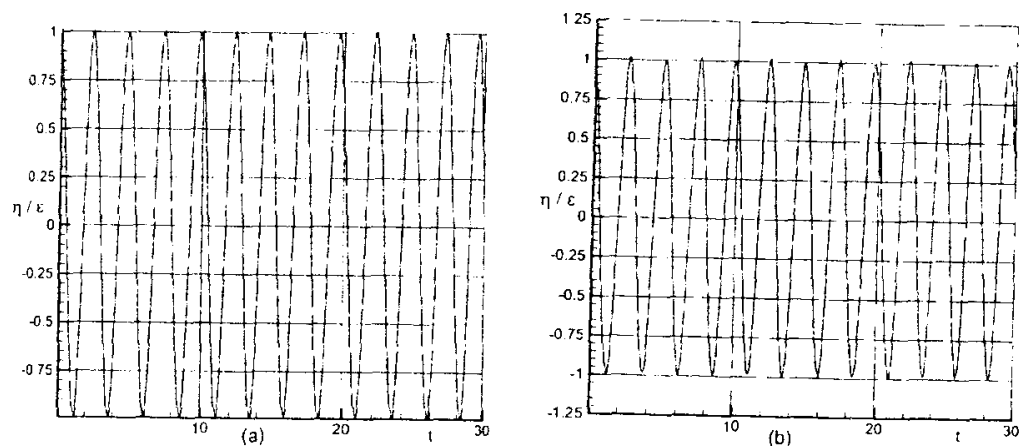

Figure 2. Comparison of the nonlinear modal solution (solid line) with the linearized solution (dashed line) for the symmetric initial value problem for the interface. The figure shows the oscillations in time of the midpoint of the liquid surface when it is initially made up of the first symmetric, linear mode alone. $\beta=0.001, h=1.5$. (a) $\varepsilon=0.002$, (b) $\varepsilon=0.004$
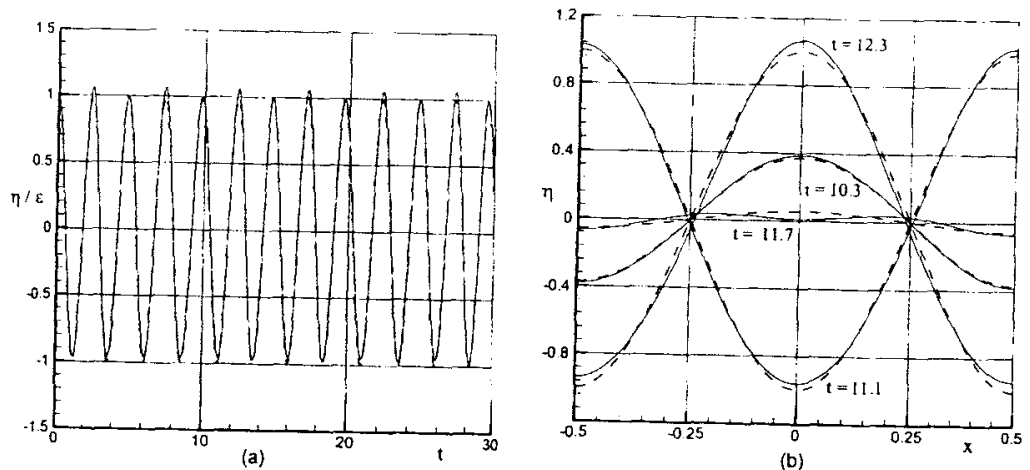

Figure 3. Comparison of the nonlinear modal solution (solid line) with the linearized solution (dashed line) as in figure 2. $\beta=0.001, h=1.5, \varepsilon=0.01$. (a) The oscillations of the midpoint of the free surface, (b) the free surface shape at different times over a cycle. 
is apparent at both peaks and troughs, the excursions are alwavs upwards. a characteristic of these nonlinear waves. Note however that the basic frequencies are almost exactly equal over more than 10 oscilation periods. The shape of the free surface at four times in a period are shown in figure $3 \mathrm{~b}$. Although the qualitative features are similar there are discernable differences between the linearized and nonlinear waveforms. It may be observed that the former has genuine nodes while the latter does not. Note also that the former is time periodic while the nonlinear waveform is not.

A question that naturally arises in the use of the modal method is that of the number of modes required to generate an accurate solution. As pointed out earlier the nature of the initial data bears on the answer to this question since $N$ has to be greater than the number of modes required to accurately represent the initial data. Given this constraint, whereas in principle increasing $N$ will lead to greater and greater accuracy, in practice we choose $N$ sufficiently large to capture the phenomena of interest and then check with still larger $N$ to make sure that we have reasonable accuracy. Whereas $3-5$ modes would be ample for the data of figure 2. the figure actually uses 10 . It is generally true, as might be expected, that with increasing amplitude more modes are required for a given accuracy over a given time period and that for large times the solution will at least drift in phase from the exact solution. The latter statement is true for all numerical calculations of the sloshing phenomenon. Unless otherwise stated all the data reported here have been obtained using 5-20 modes. It had been pointed out earlier that $a_{0}(t)$, the mean value of $\eta(x, t)$, should be zero and so the departure from this is a measure of inaccuracy of the solution. For example in figure 2 at $t=30, a_{0}$ is about $-6.2 \times 10^{-7}$ and $-2.5 \times 10^{-6}$ respectively, while at the same time in figure 3 it is about $-1.6 \times 10^{-5}$; however if $\Delta t$, the integration time step, is reduced from $10^{-3}$, the value used for figures 2 and 3 , to $10^{-4}, a_{0}(30)$ falls to $-1.6 \times 10^{-6}$ but with no perceptible effect on figure 3 . We should also point out that, like all computational schemes, this method fails in certain regions of the relevant parameter space. Stability problems are encountered generally for large values of $\varepsilon, \beta$ and $N$ and very small values of $h$. In the case of free oscillations, the total energy can be monitored as another check on the accuracy of the results. The nondimensional energy density is given by

$$
E=\frac{1}{2} \int \phi \nabla \phi \cdot \mathrm{d} S+\frac{1}{2} \int_{-1 / 2}^{1 / 2} \eta^{2} \mathrm{~d} x+\frac{1}{B o} \int_{-1 / 2}^{1 / 2} \sqrt{1+\eta_{x}^{2}} \mathrm{~d} x
$$

the three terms being the kinetic, potential and surface energies respectively. The integration in the first term is over the solid boundaries and the interface. For the case presented in figure 3 , the relative error in the energy is $2.4 \times 10^{-5}$ at $t=30$ with the relatively large time step of $t=0.01$

We would now like to demonstrate a feature that results from capillarity at the liquid free surface. Figure 4 shows the interface shapes for two large values of $\varepsilon$ and a number of times when surface tension is absent. The times are towards the end of the first period leading to the first central maximum. As $\varepsilon$ increases the wave form is more and more peaky and the departures from linearity are very large. Typical of these nonlinear sloshing motions, the excursions are always upwards. In figure 5 are shown the interface wave forms at 3 sets of times for the same parameters as in figure $4 \mathrm{a}$ but with $\beta=0.001$, i.e., with capillary effects included. Clearly visible now are capillary waves riding on the main wave form. Note that figures $4 \mathrm{a}$ and $5 \mathrm{a}$ are similar as they correspond to peaks in the central maximum; figure $5 \mathrm{~b}$ shows the situation at a minimum of the central maximum, while figure $5 \mathrm{c}$ is at an
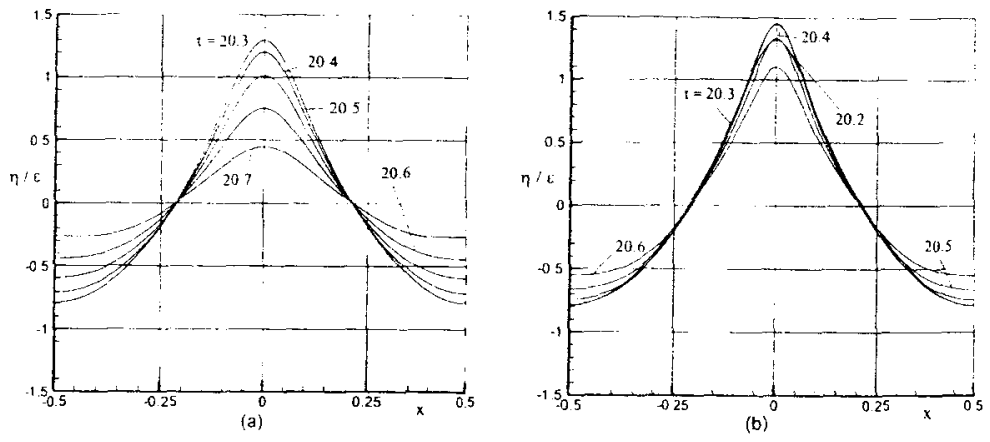

Figure 4. The free surface shapes in the absence of surface tension for comparatively large initial amplitudes. Initial shape as in figure 2. $\beta=0, h=1.5$. (a) $\varepsilon=0.04$, (b) $\varepsilon=0.05$.
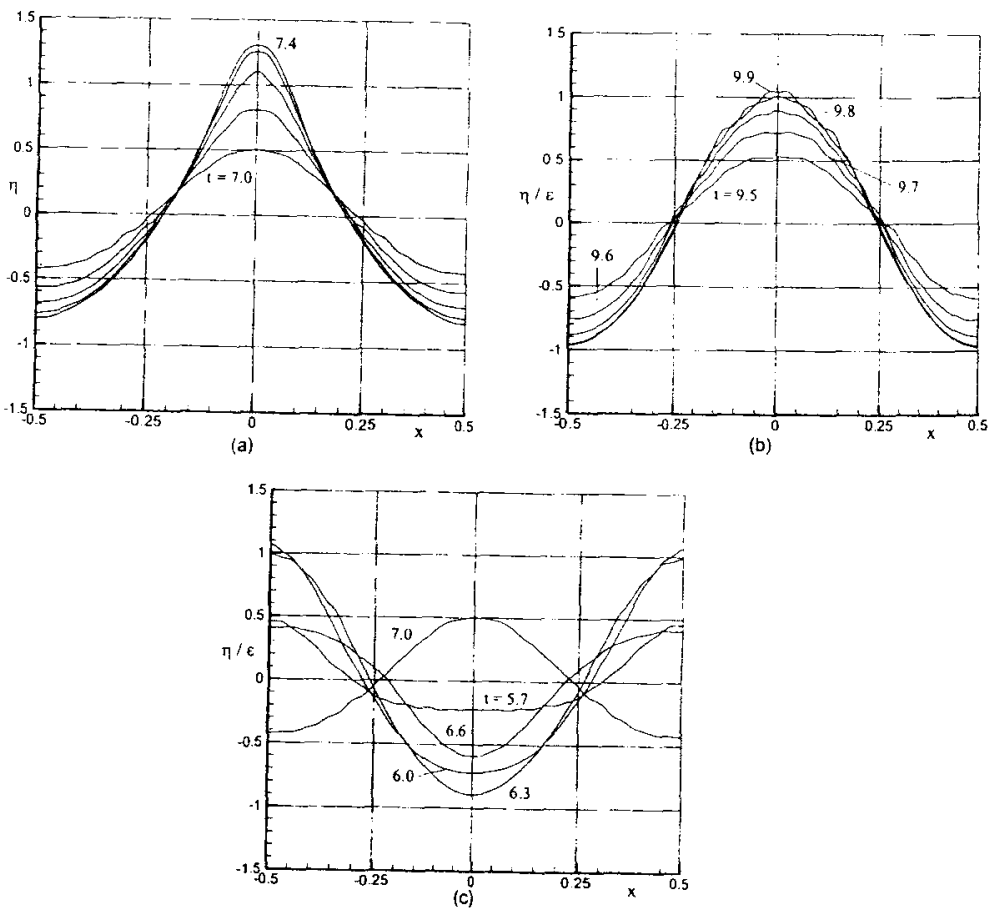

Figure 5. The generation of capillary waves on the free surface. Initial conditions as in figure 2 . $\beta=0.001, h=1.5, \varepsilon=0.04$. 

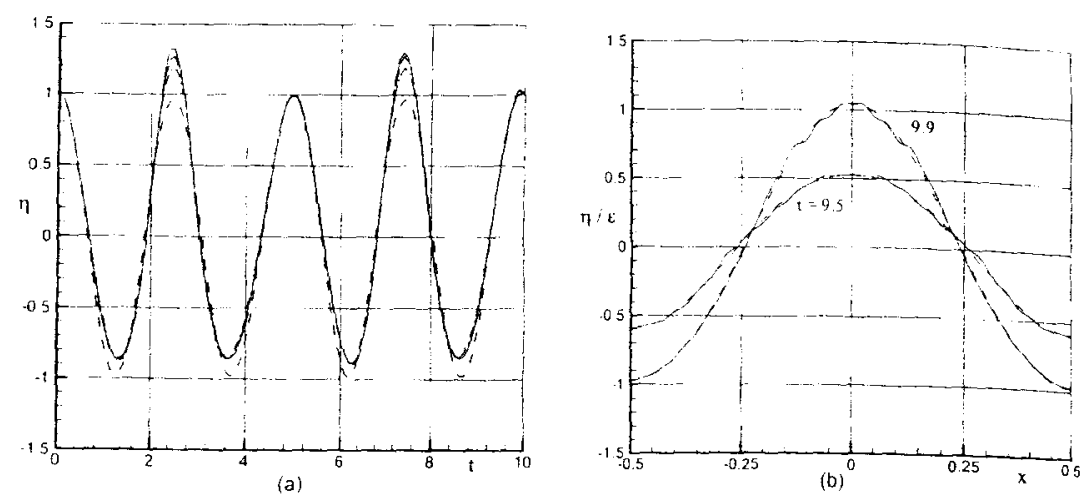

Figure 6. The lowest modes alone are not enough to describe the capillary waves seen in figure 5. This figure shows the results of using the first mode alone (long dash), using the first two modes (dash-dot), using the first three modes (short dash) and using twenty modes (solid)

actual minimum. Although capillary waves are apparent in all the frames of figure 5 there are qualitative differences: while plateaus and oscillations are seen in all of them. the peak appears to be smoother in figure 5a, while a very large plateau is seen in figure 5c. Figure 6 shows that these capillary waves cannot be captured by a method which attempts to use the first few modes alone. Whereas the overall wave form can be approximately represented by these, the capillary fine structure does require the higher modes. This is even more clearly shown in figure 7 where we have plotted the contributions of modes 10-20 alone to the wave form at various times. It is these modes that primarily contribute to the high wave number capillary waves seen in figure 5 ; the effective wave number is around $2 \pi / 17$.

Generation of such parasitic capillaries on steep gravity waves is an important and interesting topic that has been studied for the last forty years. Most of the studies have been for spatially and/or temporally periodic and steady flows. Solutions have been found, where under different conditions, ripples have been generated on the forward face of a propagating steep gravity wave (Longuet-Higgins [20,21], Jiang et al [22]), in the wave trough as in the steady solution of Schwartz and Vanden-Broeck [23] and symmetric ripple formation near the crest (Schultz et al [24]). The modal approach as presented in this paper can capture many of these details. The present calculation, with an initial symmetric profile leads to symmetric ripple formation and flattening of the crest, features that have been observed in experiments on steep Faraday waves (Schultz et al [24]). An illuminating discussion on the topic can be found in [25].

The effects of increasing capillarity are not uniform and are not easily predictable. This is shown in figure 8 , where for $\varepsilon=0.03$, the effects of increasing the surface tension or $\beta$ by three orders of magnitude, is shown. When $\beta=0.01$, the time trace (figure 8 a) shows a large subharmonic modulation caused by the large amplitudes assumed by the higher modes closest to the primary. This is borne out by figure 9 where instead of small amplitude capillary waves riding on the primary wave, the primary wave form itself is modified. When $\beta$ is increased further as in figures $8 \mathrm{~b}$ and $8 \mathrm{c}$ to 0.1 and 1.0
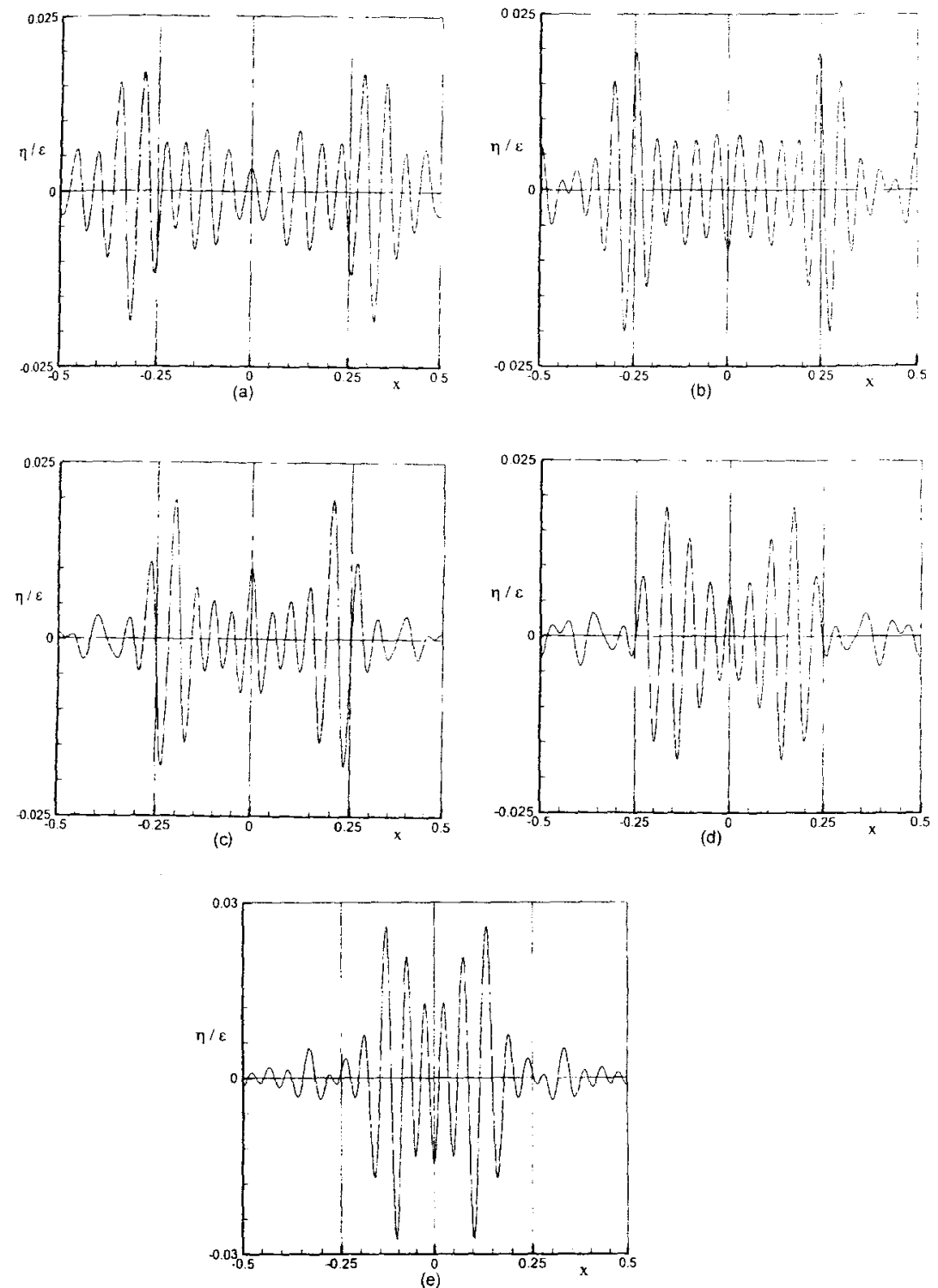

Figure 7. This figure shows that the ${ }^{*}$ capillary waves of figure 5 are contained mostly in modes 10 to 20 . These five frames show the contribution of modes 10-20 to the free surface at five different times. (a) $t=9.9$, (b) $t=9.8$, (c) $t=9.7$, (d) $t=9.6$ and (e) $t=9.5$. 

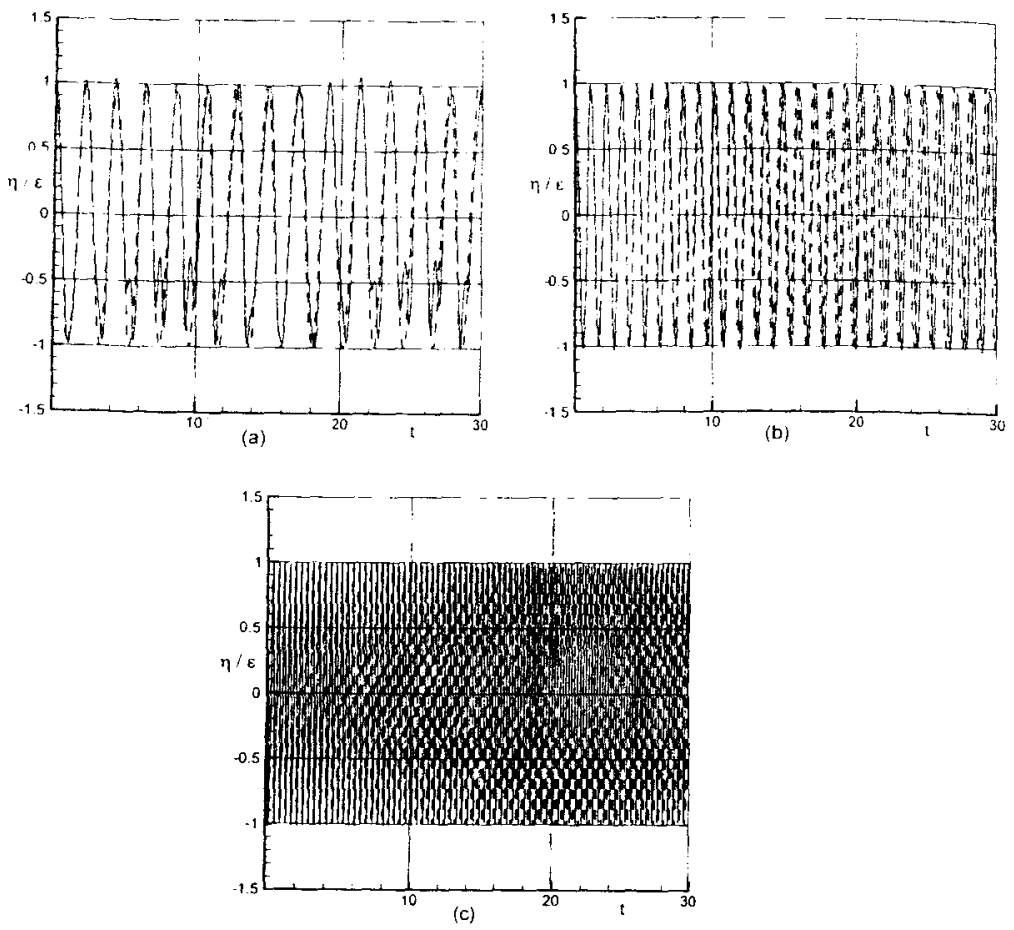

Figure 8. The effect of increasing surface tension on the free surface oscillations considered in figure 2 . $h=1.5, \varepsilon=0.03$. (a) $\beta=0.01$, (b) $\beta=0.1$, (c) $\beta=1$

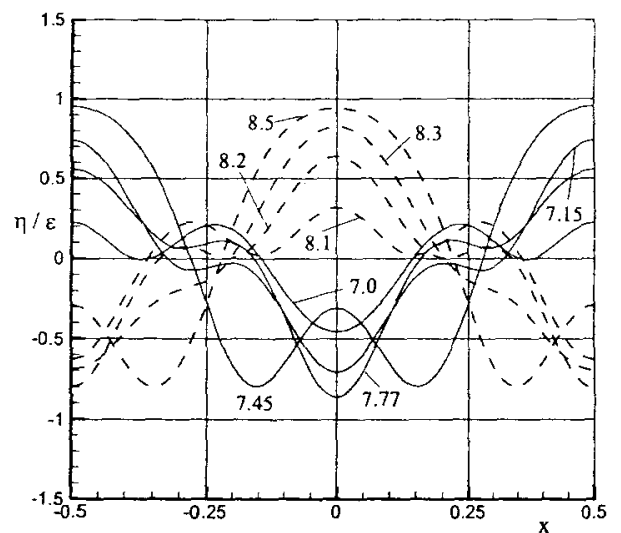

Figure 9. The large contribution of the lower modes to the free surface displacement for moderate values of the surface tension. $\beta=0.01, h=1.5, \varepsilon=0.03$. the picture actually simplifies. Recall that the linearized frequencies $\omega_{l n}$ are given by $\omega_{l n}=\sqrt{ }\left\{2 n \pi\left(1+\beta(2 n \pi)^{2}\right) \tanh 2 n \pi h\right\}$. Thus for the cases considered here the fundamental periods are approximately $2.96,1.26$ and 0.4 respectively and the rapid increase in the frequency of the uscillations is clearly seen in the figure. Now however two effects are apparent. Although with increasing $\beta$ one might expect the nonlinear curvature term to play a more prominent role, the transfer of energy to the higher modes actually decreases and the nonlinear motion greatly resembles the linear motion but with a phase shift and slight modulation. The other effect is that the excursions of the peak amplitudes seen in figures $3-6$ are absent. This is understandable as the interface acts increasingly as a tight membrane and restricts the nonlinear excursions from taking place. A surprising feature is that the small excursions that do take place are at the minima rather than at the maxima, although these are very small

In figure 9 we display the interface shape for a number of times in a cycle for the in teresting case considered in figure $8 \mathrm{a}$. In this case sufficient energy has been pumped in from the primary mode to the next higher modes that there are times when the first three modes have comparable amplitudes. The wavelengths of the first three modes are 1,1/2 and $1 / 3$ respectively and these contributions can be seen in the figure. It may be noted that the nonuniform motion of the central point on the interface only occurs on the down ward cycle in this case. Although we do not show it here, when $\varepsilon$ is increased, these nonuniform motions also occur on the upward part of the cycle.

\section{Almost periodic stationary waves}

A question that has intrigued many workers and led to a large body of classical results is whether nonlinear, finite amplitude, time periodic gravity waves exist. Although in linearized theory every individual mode is time periodic, it not at all obvious that such nonlinear waves exist. For the comparatively simpler case of spatially periodic travelling waves, where in a frame moving with the waves the motion can be considered to be stationary, the basic results were obtained by Stokes [7]; the mathematical proof of existence of such waves was given by Levi-Civita [26]. On the other hand, far less has been done for stationary gravity waves and as far as we know there is still no mathematical existence proof available for this case. In the stationary case for an infinitely deep ocean the earliest, most direct and among the most complete results are those of Penney and Price [1]. They wrote down expressions for $\eta$ and $\phi$ that are very similar to the ones that we have used. They then made use of a method of successive approximation, carrying out the calculations up to 5 th order, thereby being in a position to make an estimate of the wave of greatest height. Their work did not account for capillary effects.

What we wish to do in this section is to compare our results with the classical results of Penney and Price [1] and also with those of Tadjbakhsh and Keller [2] who also deal with stationary, periodic waves. Although the method that has been described in $\$ 2$ is incapable of systematically generating periodic solutions on its own, it is fully capable of checking a purported periodic solution if the initial data are available. What will be done here is to take the formulae for the initial values for $\eta$ and $\phi$ from the above mentioned papers and use them to generate initial data for our computational procedure; it will then be possible to compare our results with those of the earlier workers. For the convenience of the reader these formulae have been written down in the appendix to this paper. In figure 10 are compared the oscillations in time of the central point on the liquid surface as given 
by the two computations. In figure $10 \mathrm{a}$, for $\varepsilon_{p}=0.1$, the results are indistiguishable for $0<t<30$; moreover the motion appears to be periodic to this level of accuracy. However in figure $10 \mathrm{~b}$, for $\varepsilon_{p}=0.4$, small discrepancies can be made out for $t>10$. In both cases we see the typically nonlinear feature of only positive excursions of the maxima and minima about the linear values. The larger amplitude case is considered again in figure 11 where the actual interface shapes at a number of times are compared; the agreement is very good. What these figures show is that the Penney and Price [1] result is, as might be expected an excellent represention of a nearly periodic stationary wave. It is by construction exactly periodic to order 5 in their expansion. However, it cannot be exactly periodic to all orders and so we should expect departures for large amplitudes and large times and this is exactly what is found.
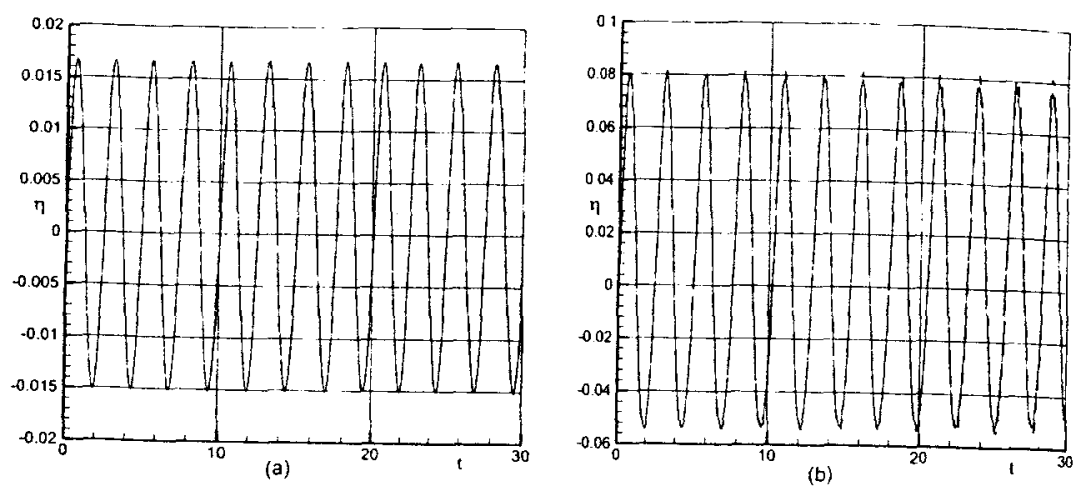

Figure 10. Comparison of the results of the present modal calculation (solid line) with those of Penney and Price [1] (dashed line). $\beta=0, h=\infty$. (a) $\varepsilon_{p}=0.1$, (b) $\varepsilon_{p}=0.4$

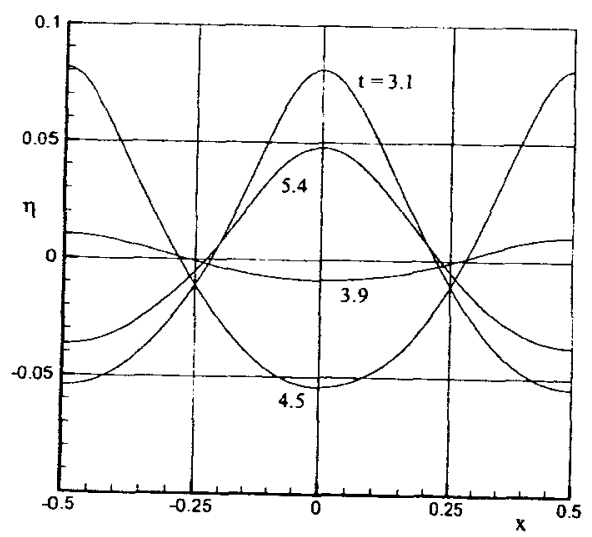

Figure 11. Comparison of the results of the present modal calculation (solid line) with those of Penney and Price $[1]$ (dashed line). $\beta=0, h=\infty, \varepsilon_{p}=0.4$.
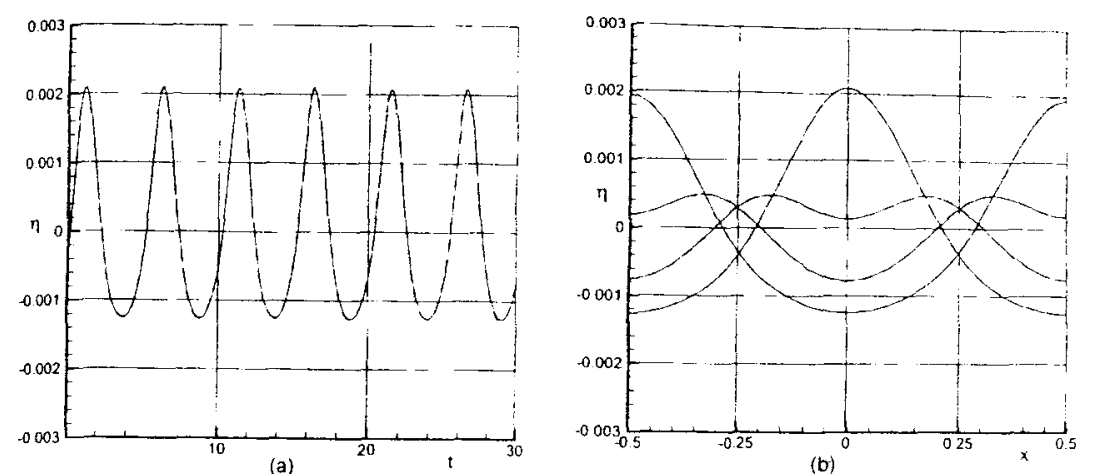

Figure 12. Comparison of the results of the present modal calculation (solid line) with those of Tadjbakhsh and Keller [2] (dashed line). $\beta=0, h=0.03978, \varepsilon_{p}=0.01$.

Tadjbakhsh and Keller [2] consider the same problem but in attempting to solve it they use a direct perturbation expansion in the initial amplitude of the first mode; they carry through their calculations up to the $3 \mathrm{rd}$ order in the expansion parameter. They also show that to that order their results agree with those of Penney and Price [1] when the fluid is of infinite depth. The intriguing aspect of Tadjbaksh and Keller [2] is that they present results for very shallow depths, when $h$ is less than $4 \%$ of the container width! This constitutes a stiff test for any method of computation. First, for a moderate amplitude $\varepsilon_{p}$ of 0.01 we compare our results with theirs in figure 12. Comparing figure 12a with figure 10, we note that apart from the positive excursions of the extrema, we here see a clear narrowing of the peaks compared to the valleys. The approximation to periodicity is excellent as is the comparison between the two calculations. However, the situation is very different when $\varepsilon_{p}$ is increased to the much larger value of 0.05 , the value used in figures 1 and 2 of Tadjbakhsh and Keller [2]. Figure 13 shows that the 3rd order result of Tadjbakhsh and Keller is quite inadequate to handle such a large disturbance amplitude. Not only is the departure from periodicity considerable but there are large discrepancies, even qualitative, between the perturbation solution and the modal calculation using the same initial data. We must conclude that this set of parameters is outside the range where the perturbation solution is valid.

\section{Sloshing in a linearly accelerated container}

In this section, we study sloshing motions in a container undergoing sinusoidal accelerations in the $x$-direction. Unlike in the symmetric sloshing case of $\$ 3$, here we need the full representation (2a,b) for $\eta(x, t)$ and $\phi(x, y, t)$. This is because (a) the acceleration term is anti-symmetric and (b) the anti-symmetric modes generate the symmetric ones.

We assume zero initial conditions, i.e., $\eta(x, 0)=0$ and $\phi(x, y, 0)=0$. The acceleration is assumed to be given by $A_{x}(t)=-\varepsilon \omega_{f}^{2} \sin \omega_{f} t$ where $\varepsilon$ is the displacement amplitude of the container. 

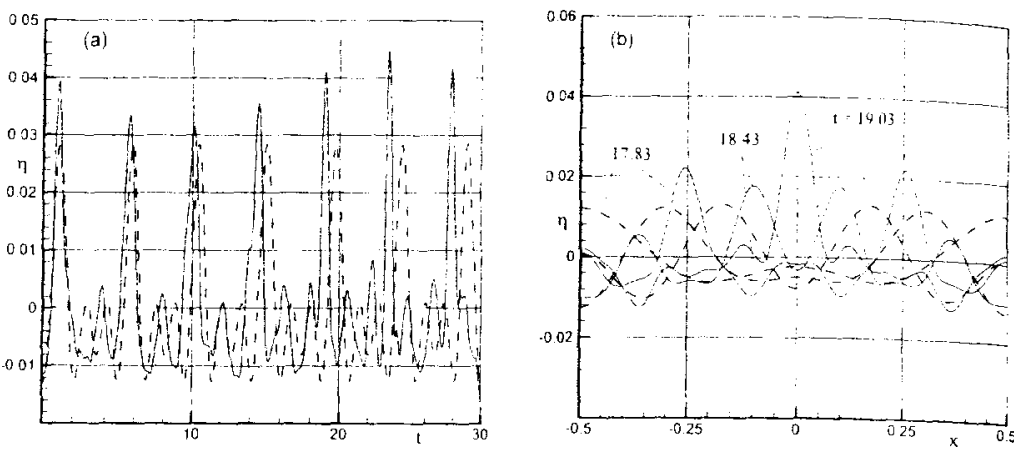

Figure 13. Comparison of the results of the present modal calculation (solid line) with those of Tadjbakhsh and Keller [2] (dashed line). $\beta=0 . h=0.03978, \varepsilon_{n}=0.05$. It is clear that the perturbation calculation is invalid for such a large value of $\varepsilon$
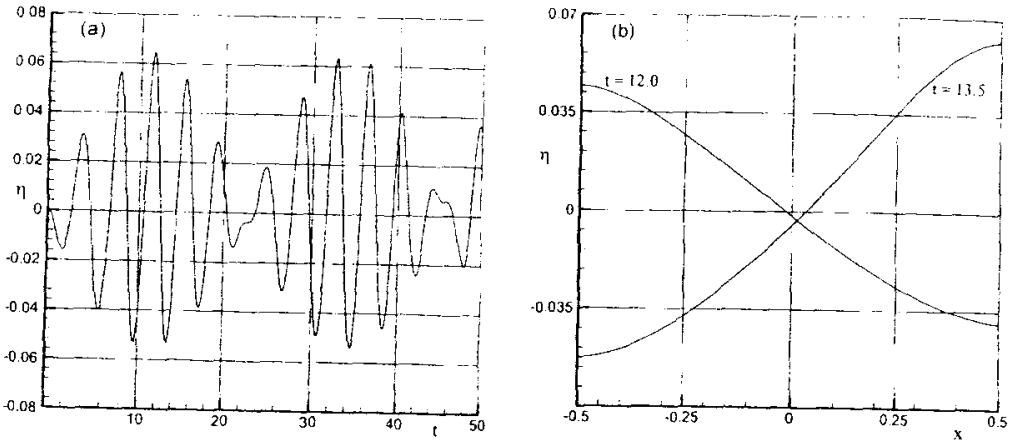

Figure 14. Sloshing in a linearly accelerating container. $\varepsilon=0.01, \omega_{f}=1.5, \beta=0.001$ (a) Oscillations in time of the point on the interface at the left wall. (b) The free surface shapes, at two arbitrary times, showing asymmetry.

Figure 14a shows the time trace of the point on the interface at the left wall when $\varepsilon=0.01, \omega_{f}=1.5$ and $\beta=0.001$. A modulation of the envelope is clearly visible with modulation frequency approximately equal to the difference between the first odd natural linear frequency, 1.781 and the driving frequency, $\omega_{f}$. Figure $14 \mathrm{~b}$ shows the free surface at two different instants of time and it is seen that the initially flat interface evolves to neither a symmetric nor an anti-symmetric shape but to one which is a mixture of both types of modes.

Finally, we present some comparisons with the results of Faltinsen et al [3] who have studied sloshing in a linearly accelerating container both experimentally and through numerical computations using a three mode nonlinear system. Figure 15 shows the time trace at $x=-0.47$. Both axes have been suitably rescaled for direct comparison. This compares very favorably with tigure 6 of Faltinsen et al [3]. The present computation is performed with only two modes.

We now discuss a case of shallow water, where due to secondary parametric resonance, the first mode is not dominating. Figure 16 show: the time trace at the left wall for one such case. The parameters are $\varepsilon=0.01$ ( (1) $=1.19935$ and $h=0.1734$. The solid line is the

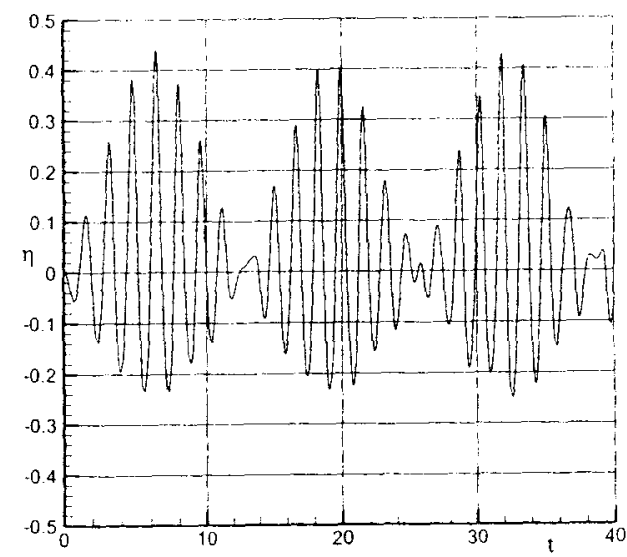

Figure 15. Comparison with Faltinsen et al [3]. Oscillations in time of the point $x=$ -0.47 on the interface. Excellent agreement with figure 6 of Faltinsen et al [3] is observed.

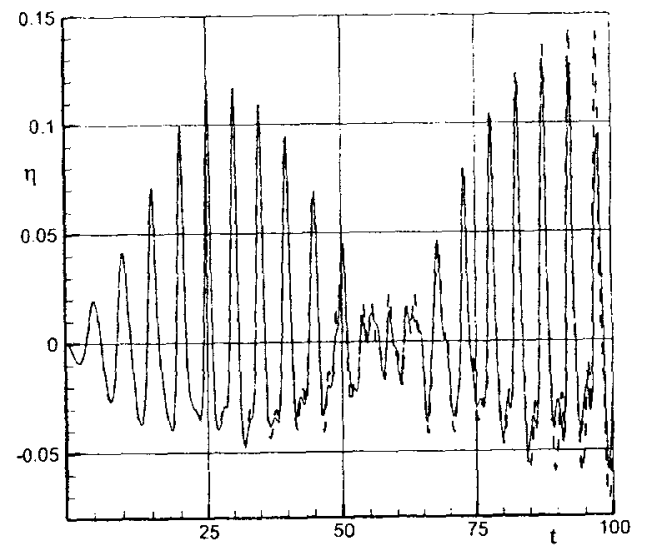

Figure 16. Sloshing in a linearly accelerating container-shallow water case. $\varepsilon=$ $001, \omega_{f}=1.19935, h=0.1734$. Oscillations in time of the point on the interface at the left wall using three (dashed) and four (solid) modes. 
result of using four modes in the computation, the dashed line three. The two are in very good agreement over most of the first cycle though later on, there are clear differences. The crest-to-trough amplitude is roughly 15 times the displacement amplitude of the container.

\section{Conclusion}

We have suggested in this paper a method for calculating the sloshing motion of an ideal liquid in a rectangular container. Although the use of modes for solving the linearized problem is classical and even their occasional use for tackling the nonlinear case is known, we believe that the present implementation is new. Its main advantage is its simplicity and generality. We have shown this by solving a number of difficult problems that were considered by earlier authors and by displaying a wide range in applications of the method over the range of parameters. Moveover, some surprising new results, especially on the effects of capillarity, have been displayed using this powerful tool. This method can handle situations which require the use of a large number of modes. We conclude by pointing out that the method can be extended to other simple geometries where modes arise simply and naturally.

\section{Appendix}

We present here the formulae of Penney and Price [1] and Tadjbaksh and Keller [2], that were used in $\$ 4$ to generate initial data for determining the periodic wave. Since the normalizations in $[1,2]$ are different from ours, we first give the relations between their variables (hatted) and ours. The relations are $(\hat{x}, \hat{y}, \hat{h})=2 \pi(x, y, h), \hat{\phi}=\frac{(2 \pi)^{3 / 2}}{\varepsilon} \phi, \hat{\eta}=\frac{2 \pi}{\varepsilon} \eta$ and $\hat{t}=\omega t$.

There is an additional negative sign on the RHS of the $\phi$ equation in the case of Penney and Price [1], with the other relations remaining unchanged. We now give the formulae for $\eta$ and $\phi$ from [1], written in our nondimensional variables. These are

$$
\eta(x, t)=\frac{1}{2 \pi} \sum_{n=1}^{5} a_{n}(t) \cos 2 n \pi x
$$

where

$$
\begin{aligned}
a_{1}= & \left(\varepsilon+\frac{3}{32} \varepsilon^{3}-\frac{137}{3072} \varepsilon^{5}\right) \sin \omega t+\left(\frac{1}{16} \varepsilon^{3}-\frac{11}{5376} \varepsilon^{5}\right) \sin 3 \omega t \\
& +\frac{163}{21504} \varepsilon^{5} \sin 5 \omega t \\
a_{2}= & \frac{1}{4} \varepsilon^{2}+\frac{1}{16} \varepsilon^{4}-\left(\frac{1}{4} \varepsilon^{2}-\frac{25}{192} \varepsilon^{4}\right) \cos 2 \omega t-\frac{67}{1344} \varepsilon^{4} \cos 4 \omega t \\
a_{3}= & \left(\frac{9}{32} \varepsilon^{3}-\frac{1}{256} \varepsilon^{5}\right) \sin \omega t-\left(\frac{3}{32} \varepsilon^{3}-\frac{2195}{14336} \varepsilon^{5}\right) \sin 3 \omega t \\
& -\frac{16365}{473088} \varepsilon^{5} \sin 5 \omega t,
\end{aligned}
$$

$$
\begin{aligned}
& a_{4}=\left(\frac{1}{8}-\frac{1}{6} \cos 2 \omega t+\frac{1}{24} \cos 4 \omega t\right) \varepsilon^{4} \\
& a_{5}=\left(\frac{145}{768} \sin \omega t-\frac{515}{3072} \sin 3 \omega t+\frac{85}{3072} \sin 5 \omega t\right) \varepsilon^{5}
\end{aligned}
$$

and

$$
\omega=\sqrt{ } 2 \pi\left(1-\frac{1}{4} \varepsilon^{2}-\frac{13}{128} \varepsilon^{4}\right)^{1 / 2} .
$$

Thus, the initial condition for $\eta$ is given by

$$
\eta(x, 0)=\frac{1}{14 \pi} \varepsilon^{4} \cos 4 \pi x .
$$

The initial condition for $\phi$ is given by

$$
\phi(x, y, 0)=\frac{1}{(2 \pi)^{3 / 2}} \sum_{n=1}^{5} \beta_{n} \cos 2 n \pi x \mathrm{e}^{2 n \pi y}
$$

where

$$
\begin{aligned}
& \beta_{1}=\varepsilon+\frac{5}{32} \varepsilon^{3}-\frac{252}{7168} \varepsilon^{5} \\
& \beta_{2}=0, \quad \beta_{3}=\frac{48}{2112} \varepsilon^{5} \\
& \beta_{4}=0
\end{aligned}
$$

and

$$
\beta_{5}=\frac{72}{2048} \varepsilon^{5} .
$$

In the case of Tadjbaksh and Keller [2], the formulae are

$$
\begin{aligned}
\eta(x, t)= & \frac{\varepsilon}{2 \pi} \sin \omega t \cos 2 \pi x+\frac{\varepsilon^{2}}{16 \pi}\left(\omega_{0}^{2}+\omega_{0}^{-2}\right. \\
& \left.+\left(\omega_{0}^{-2}-3 \omega_{0}^{-6}\right) \cos 2 \omega t\right) \cos 4 \pi x \\
& +\frac{\varepsilon^{3}}{4 \pi}\left(b_{11} \sin \omega t \cos 2 \pi x+b_{13} \sin \omega t \cos 6 \pi x\right. \\
& \left.+b_{31} \sin 3 \omega t \cos 2 \pi x+b_{33} \sin 3 \omega t \cos 6 \pi x\right)
\end{aligned}
$$

where

$$
\begin{aligned}
& \omega=\sqrt{2 \pi}\left(\omega_{0}+\frac{\varepsilon^{2}}{2} \omega_{2}\right), \\
& \omega_{0}^{2}=\tanh 2 \pi h, \\
& \omega_{2}=\frac{1}{32}\left(9 \omega_{0}^{-7}-12 \omega_{0}^{-3}-3 \omega_{0}-2 \omega_{0}^{5}\right),
\end{aligned}
$$




\section{P N Shankar and R Kidambi}

$$
\begin{aligned}
& b_{11}=\frac{1}{32}\left(3 \omega_{0}^{-8}+6 \omega_{0}-4-5+2 \omega_{0}^{4}\right) \\
& b_{13}=\frac{3}{128}\left(9 \omega_{0}^{-8}+27 \omega_{0}^{-4}-15+\omega_{0}^{4}+2 \omega_{0}^{8}\right) \\
& b_{31}=\frac{1}{128}\left(3 \omega_{0}^{-8}+18 \omega_{0}^{-4}-5\right)
\end{aligned}
$$

and

$$
b_{33}=\frac{3}{128}\left(-9 \omega_{0}^{-12}+3 \omega_{0}^{-8}-3 \omega_{0}^{-4}+1\right) .
$$

Thus, the initial condition for $\eta$ becomes

$$
\eta(x, 0)=\frac{\varepsilon^{2}}{16 \pi}\left(\omega_{0}^{2}+2 \omega_{0}^{-2}-3 \omega_{0}^{-6}\right) \cos 4 \pi x .
$$

The initial condition for $\phi$ is given by

$$
\begin{aligned}
\phi(x, y, 0)= & \frac{\varepsilon}{(2 \pi)^{3 / 2}}\left[\omega_{0} \cos 2 \pi x \frac{\cosh 2 \pi(y+h)}{\sinh 2 \pi h}\right. \\
& \left.+\frac{\varepsilon^{2}}{2}(\alpha \cos 6 \pi x \cosh \pi(y+h)+\beta \cos 2 \pi x \cosh 2 \pi(y+h))\right]
\end{aligned}
$$

where

$$
\alpha=\frac{1}{128 \cosh 6 \pi h}\left(1+3 \omega_{0}^{4}\right)\left(-9 \omega_{0}^{-13}+25 \omega_{0}^{-9}-13 \omega_{0}^{-5}-5 \omega_{0}^{-1}+2 \omega_{0}^{3}\right)
$$

and

$$
\beta=\frac{1}{128 \cosh 2 \pi h}\left(9 \omega_{0}^{-9}+62 \omega_{0}^{-5}-31 \omega_{0}^{-1}\right)
$$

\section{References}

[11 W G Penney and A T Price, Philos. Traps. A244,254 (1952)

[2] 1 Tadjbakhsh and J B Keller, J. Fluid Mech. 8,442 (1960)

[3] O M Faltinsen, O F Rognebakke. I A Lukovsky and A N Timokha, J. Fluid Mech. 407, 201 (2000)

[4] V E B Dussan, Ann. Rev. Fluid Met it. 11, 371 (1979)

[5j L M Hocking. J. Fluid Mech. 179. 253 (1987)

[6] H Lamb, Hvdrodwtmnics (Cambridge University Press, 1932)

[7] G G Stokes, Collected papers 5. 62 (1880)

[8] P Concur, J. Fluid Mech. 14,568 (1962)

[9] L W Schwartz and A K Whitney, J. Fluid Mech. 107. 147 (1981)

[10] G D Crapper. J. Fluid Mech. 2. 532 (1957)

111 J R Ockendon and H Ockendon. J. Fluid M ch. 59. 397 (1973)

[12] J W Miles. J. Fluid Mech. 146, 285 (1984)

[1 3] M Nagata, J. Fluid Mech. 209, 265 (1989)

\section{A modal method for finite amplitude, nonlinear sloshing}

[14] L Shemer. J. Fluid Mech. 217, | 43 (1990)

[15] M Umeki, J Fluid Mech. 227, 161 (1991)

[16] P Mclver, J. Fluid Mech. 201, 243 (1989)

[17] R E Moore and L M Perko, J. Fluid Mech. 22, 305 (1964)

[18] L M Perko, J. Fluid Mech. 35, 77 (1969)

[19] M La Rocca, G Sciortino and M A Boniforti. Fluid Dyn. Res. 27, 23 (2000)

[201 M S Longuet-Higgins, J. Fluid Mech. 16, 138 (1963)

[21] M S Longuet-Higgins, J. Fluid Mech. 301, 79 (1995)

[22] L Jiang, H-J Lin, W W Schultz and M Perlin, J. Fluid Mech. 386, 281 (1999)

[23] L W Schwartz and J-M Vanden-Broeck, J. Fluid Mech. 95, 119 (1979)

[24] W W Schultz, J-M Vanden-Broeck, L Jiang and M Perlin, J. Fluid Mech. 369, 253 (1998)

[25] M Perlin and W W Schultz, Ann. Rev. Fluid Mech. 32, 241 (2000)

[26] T Levi-Civita, Math. Ann. 93, 264 (1925) 